OPEN ACCESS

Edited by:

Thomas FitzGerald,

University of Massachusetts Boston,

United States

Reviewed by:

A. Nasser Khalifeh

University of Massachusetts Medical

School, United States

Fenghong Liu,

UMass Memorial Medical Center

United States

*Correspondence:

Tao Zhou

zt1974sd@163.com

Qing Kong

qkong@fudan.edu.cn

Zhiyong Xu

xzyong12vip@sina.com

Specialty section:

This article was submitted to

Radiation Oncology,

a section of the journal

Frontiers in Oncology

Received: 22 September 2021

Accepted: 25 October 2021

Published: 15 November 2021

Citation:

Duan $Y$, Lin $Y$, Wang $H$ Kang $B$, Feng $A, M a K$, Chen $H$, Huang Y, Gu H, Shao Y, Zhou T, Kong $Q$ and $X u Z$ (2021) How Does

the Gradient Measure of the Lung

SBRT Treatment Plan Depend on the Tumor Volume and Shape?

Front. Oncol. 11:781302.

doi: 10.3389/fonc.2021.781302

\section{How Does the Gradient Measure of the Lung SBRT Treatment Plan Depend on the Tumor Volume and Shape?}

\author{
Yanhua Duan ${ }^{1}$, Yang Lin ${ }^{1}$, Hao Wang ${ }^{1}$, Bodong Kang ${ }^{2}$, Aihui Feng ${ }^{1}$, Kui Ma ${ }^{3}$, Hua Chen ${ }^{1}$, \\ Ying Huang ${ }^{1}$, Hengle $\mathrm{Gu}^{1}$, Yan Shao ${ }^{1}$, Tao Zhou ${ }^{4 *}$, Qing Kong ${ }^{5^{*}}$ and Zhiyong $\mathrm{Xu}^{{ }^{1 *}}$ \\ ${ }^{1}$ Department of Radiation Oncology, Shanghai Chest Hospital, Shanghai Jiao Tong University, Shanghai, China, \\ 2 Pekoe Team, MIM Software Inc., Cleveland, OH, United States, ${ }^{3}$ Clinical Helpdesk, Varian Medical Systems, Beijing, China, \\ ${ }^{4}$ Shandong Cancer Hospital and Institute, Shandong First Medical University and Shandong Academy of Medical Sciences, \\ Jinan, China, ${ }^{5}$ Institute of Modern Physics, Fudan University, Shanghai, China
}

Purpose: Gradient measure (GM) is a critical index related to normal tissue sparing in radiosurgery. This study aims to describe the dependence of GM on target volume and target shape for lung stereotactic body radiation therapy (SBRT) treatment plans.

Methods: A total of 307 peripheral and 119 central lung SBRT treatment plans were enrolled for this study. A least-squares regression was used for data analysis. First, the equations with different functional forms were established to determine the dependence of GM on a univariaty $\left(V_{P}\right.$ or $\left.S p\right)$ and bivariaty $\left(V_{P}\right.$ and $\left.S p\right)$, respectively. Then, the correlation coefficients and $p$-values of variables for all equations were compared and analyzed to determine the dependence of GM on PTV volume (VP) and sphericity (Sp).

Results: The power equations had the highest coefficient of determination $\left(R^{2}\right)$ in the dependence results of $\mathrm{GM}$ on univariate $\mathrm{V}_{\mathrm{P}}$. The equations were $\mathrm{GM}=0.674 \mathrm{~V}_{P}^{0.178}$ and $\mathrm{GM}=0.660 \mathrm{~V}_{P}^{0.185}$ for peripheral and central lesions, respectively. On the other hand, the $R^{2}$ of all functional forms were less than 0.25 when the relationship of GM versus univariate $S p$ was analyzed. Similarly, the power equation also obtained the highest $R^{2}$ in bivariaty $V_{P}$ and $\mathrm{Sp}$ analysis, whether for central or peripheral. However, the $\mathrm{R}^{2}$ of the bivariate equations were not improved compared with those of univariate equations. Moreover, the $\mathrm{p}$-values of the variable Sp were greater than 0.05 .

Conclusions: The GM of the lung SBRT plan is shape-independent and volumedependent. The dependence of GM on PTV volume for peripheral and central lung cancer can be described by two different power equations. The results of this study can be used as a potential tool to assist dosimetric quality control during the radiosurgery process.

Keywords: SBRT, lung cancer, radiotherapy, gradient measure, volume, shape 


\section{INTRODUCTION}

As a technique that has been widely employed, stereotactic body radiotherapy (SBRT) can be used to deliver high fractional dose in few fractions. Compared with traditional radiotherapy, SBRT provides better efficacy, lower toxicity, and shorter treatment duration $(1,2)$. Clinical evidence and studies have shown that the therapeutic effect of early non-small cell lung cancer (NSCLC) patients treated with SBRT is similar to or even better than that of surgery $(1,2)$ and that SBRT has become a major alternative therapy for patients with NSCLC who are unsuitable or unwilling to undergo surgery (3-5).

In order to achieve a therapeutic effect similar to surgery, a highly conformal SBRT treatment plan needs to give the tumor high-dose precise ablation while minimizing organs at risk (OARs) damage, which requires a sharp dose gradient nearly isotropically around the target $(1,2,6-8)$. In clinical practice, the targets of different patients are various, and the dose gradient largely depends on the individualized geometric characteristics of the target $(7,9-13)$.

Some scholars have recently studied the dependence of the dose gradient on the target volume for SBRT plans $(7,14)$. Those studies lay a foundation for the dependence of dose gradient on target geometry in lung SBRT plan. However, most studies focused on the relationship between dose gradient and target volume, while the impact of target shape on the dose fall-off is still unclear. In addition, lung cancer patients treated with SBRT include peripheral and central types. Due to the significant difference in tumor anatomical location between those two types, the factors considered in the planning process are also different. It is unclear whether this leads to the dependence difference of dose gradient on target volume and shape between two types of lung cancer. Although RTOG has used the same criteria for $\mathrm{R} 50 \%$, which is defined as the ratio of $50 \%$ prescription isodose volume to the PTV volume characterizing the dose fall-off for both peripheral and central lesions, the difference in the location of the two types of tumors results in their different dose gradients. Therefore, it is necessary to investigate the dependence of dose gradient on target volume and shape, respectively, for peripheral and central lung SBRT plans.

In this study, a large number of clinically acceptable peripheral and central lung SBRT plans were used to analyze the dependence of dose gradient on target volume and shape. The results are to determine a definite relationship between the dose gradient and target geometry for lung SBRT plans and provide a possible tool for the prediction of dose gradient before the planning process or the quality review after optimization of a lung SBRT plan.

\section{MATERIALS AND METHODS}

The approved lung SBRT treatment plans in Shanghai Chest Hospital were retrospectively selected. When the study began, all selected patients signed informed consents and completed radiotherapy. Ethical standards and patients' confidentiality were ensured and in line with regulations of the local institutional review board and data safety laws. This study was approved by the Ethics Committee of Shanghai Chest Hospital (the committee's reference Number: KS1863).

\section{Target Delineation and Treatment Planning}

Targets and OARs were delineated on a MIM Maestro Station (MIM Vista Corp, Cleveland, US-OH) based on fourdimensional CT (4DCT) by radiation oncologists. All structures were reviewed and approved by an experienced radiation oncologist before being used for planning design. All the treatment plans were planned on the average 4DCT image using the Pinnacle Treatment Planning System (TPS) (V9.10, Philips Radiation Oncology Systems, Fitchburg, WI, USA) for an Edge $^{\mathrm{TM}}$ linear accelerator (Varian Medical Systems, Palo Alto, CA) equipped with a high-definition 120 multileaf collimator (MLC). The included treatment plans ranged from three to eight fractions, and the planning method was similar to our previous research (15). In short, treatments were planned following the guidelines of RTOG 0813 (16) or 0915 (17) depending on its tumor size, the patient's physical condition, and location, which employed the IMRT technique with 10 or more $6 \mathrm{MV}$ fields. Collimator and couch angles were adjusted according to the individual situation. The collapsed cone convolution (CCC) algorithm was used for dose calculation with a calculation resolution of $1.0 \mathrm{~mm}$.

\section{Data Extraction}

This study analyzed the dependence of gradient measure (GM) (10) on PTV volume $\left(\mathrm{V}_{\mathrm{P}}\right)$ and sphericity $(\mathrm{Sp})$.

The Eclipse (Varian, Palo Alto, CA) TPS reports GM, which is defined as the difference, in centimeters, of the equivalent sphere radii of the $50 \%$ and $100 \%$ prescription isodose line volumes ( 7 , 10). This metric can quickly assess the dose gradient and has become a helpful tool for evaluating lung SBRT plans.

GM was computed as $(8,10,18)$

$$
G M=\sqrt[3]{\frac{3 V_{50 \% R x}}{4 \pi}}-\sqrt[3]{\frac{3 V_{R x}}{4 \pi}}
$$

where $\mathrm{V}_{50 \% \mathrm{Rx}}$ and $\mathrm{V}_{\mathrm{Rx}}$ are the volumes receiving a dose equal to or greater than $50 \%$ and $100 \%$ prescription dose, respectively.

Sphericity is a parameter that characterizes the shape of a three-dimensional structure. It is defined as the quotient of the surface area of a sphere and the surface area of a structure with the same volume. Sp ranges from 0 to 1 , where $S p=1$ indicates a sphere.

Sp was calculated as

$$
S_{p}=\frac{4 \pi\left(\frac{3 V_{P}}{4 \pi}\right)^{\frac{2}{3}}}{S_{P T V}}
$$

where $S_{\text {PTV }}$ is the surface area of PTV.

It can be seen from Equations 1 and 2 that the original data used for analysis include $\mathrm{V}_{\mathrm{P}}, 50 \%$ and $100 \%$ prescription isodose 
volumes, and Sp. First, a prewritten script in Pinnacle TPS was used to convert $50 \%$ and $100 \%$ prescription isodose lines to structures, and those structures were imported into MIM Maestro Station together with PTV contour. Then, a workflow embedded in the MIM Maestro station was used to calculate and extract the above four data.

\section{Data Analysis}

The dependence of GM on $\mathrm{V}_{\mathrm{P}}$ and $\mathrm{Sp}$ was analyzed using the least-squares regression (7). All analyses were performed using SPSS 22.0 (SPSS Inc., Armonk, NY).

Firstly, curve estimation was used to analyze the dependence of $\mathrm{GM}$ on a univariaty $\left(\mathrm{V}_{\mathrm{P}}\right.$ or $\left.\mathrm{Sp}\right)$ for peripheral and central lung SBRT plans. The curve fitting included linear, logarithmic, exponential, power, and logistic functional forms. Then, the fitting equations of $\mathrm{GM}$ on bivariaty $\left(\mathrm{V}_{\mathrm{P}}\right.$ and $\mathrm{Sp}$ ) were established. The regression equations include linear (Eq. 3), nonlinear sum (Eq. 4), logarithmic (Eq. 5), exponential (Eq. 6), and power (Eq. 7) functional forms.

$$
\begin{gathered}
G M=a V_{P}+c S p+e \\
G M=a V_{P}^{b}+c S p^{d}+e \\
G M=\log _{a} V_{P}^{b} S p^{c}+d \\
G M=a b^{V_{P}} c^{S p}+d \\
G M=a V_{P}^{b} S p^{c}+d
\end{gathered}
$$

The coefficient of determination $\left(\mathrm{R}^{2}\right)$ is the standard metric for evaluating the fitting goodness between model simulations and observations. Generally, the fitting model can be considered acceptable if $\mathrm{R}^{2}$ is equal to or above 0.5 (19). The p-value of the variable can reflect the reliability of a fitting equation. The contribution of a variable to an equation is considered reliable when $\mathrm{p}<0.05$.

\section{Result Verification}

In order to verify the accuracy of the final fitting equation, we use an independent external verification set to test the results to obtain the error between the calculated GM and the actual GM. The validation set included 100 peripheral lung cancer SBRT plans and 40 central lung cancer SBRT plans.

\section{RESULTS}

\section{Details of the Enrolled Cases}

A total of 426 lung SBRT plans in our center from May 2018 to June 2021 were enrolled for this study, including 307 (72\%) peripheral and 119 (28\%) central. Central was defined as being within a $2-\mathrm{cm}$ radius of the airway or mediastinal pleura (7). For peripheral, $V_{P}$ ranged from 4.79 to $261.77 \mathrm{cc}$, and $\mathrm{Sp}$ ranged from
$74.86 \times 10^{-2}$ to $99.92 \times 10^{-2}$. For central, $\mathrm{V}_{\mathrm{P}}$ ranged from 7.76 to $144.56 \mathrm{cc}$, and $\mathrm{Sp}$ ranged from $73.51 \times 10^{-2}$ to $97.31 \times 10^{-2}$. The number distribution of the $\mathrm{V}_{\mathrm{P}}$ and $\mathrm{Sp}$ for peripheral and central lesions is shown in Figure 1. The dose constraints to the targets and OARs met the proposal of RTOG 0813 (16) or 0915 (17) guidelines in all plans. Averages of the treatment plan data binned using $V_{P}$ bins from RTOG 0813 and 0915 are listed in Table 1. Table 1 also lists the actual and analytic GM values of each group for comparison, and the two results were similar. In order to refer to RTOG metrics, we also listed the planned R50\% value in Table 1.

\section{Results of Univariate Analysis}

Table 2 lists the $\mathrm{R}^{2}$ of different dependence equations of GM on $V_{P}$ and the $p$ values of the independent variable. The power equation had the highest $R^{2}$ in all functional forms $(p<0.001)$ for two types of lung SBRT plans. The equations of GM versus $V_{P}$ were Equations 8 and 9 for peripheral and central lesions, respectively.

$$
G M=0.674 V_{P}^{0.178}
$$

with a standard error of 0.016 and 0.007 for the two parameters and an $\mathrm{R}^{2}$ value of 0.675 .

$$
G M=0.660 V_{P}^{0.185}
$$

with a standard error of 0.040 and 0.016 for the two parameters and an $\mathrm{R}^{2}$ value of 0.526 .

The improved $\mathrm{R}^{2}$ in Equation 8 shows that it can explain a greater percent of the random variation of peripheral lesions' GM than Equation 9 can explain that of central lesions.

Figure 2 presents GM versus VP scatter plots, including figures of power equations and their residuals for peripheral and central lesions. For peripheral lesions, the residuals appear to be nearly randomly distributed. Most of them are within $0.25 \mathrm{~cm}$. Equation 8 predicted a lower GM in 149 cases (48.53\%) and a greater GM in 158 cases $(51.47 \%)$ than in the clinical plans. When $V_{P}$ was greater than $125 \mathrm{~cm}^{3}$, Equation 8 consistently analyzed a higher GM than the actual value. For central lesions, the distribution of residuals also seems random. Equation 9 analyzed a lower GM in 67 cases $(56.30 \%)$ and a greater GM in 52 cases $(43.69 \%)$ than what was achieved clinically.

Figure 3 shows an example of the analytic and clinical 50\% isodose lines for peripheral and central lung SBRT plans. The analytic $50 \%$ isodose lines were generated by GM calculated using Equations 8 and 9. It can be seen from the figure that the analytic and clinical dose gradients are in good agreement, especially for the peripheral lesion.

The $\mathrm{R}^{2}$ of different fitting equations of GM versus $\mathrm{Sp}$ and $\mathrm{p}$ values of variable $\mathrm{Sp}$ are also tabulated in Table 2. Among them, the exponential equation had the highest $\mathrm{R}^{2}$, but less than 0.25 ( 0.239 for peripheral and 0.152 for central) $(\mathrm{p}<0.001)$. There was a weak correlation between the analytic and clinical results. The fitting equation was unacceptable, which signifies that the GM has little dependence on the shape variable Sp for both peripheral and central lung SBRT plans. 

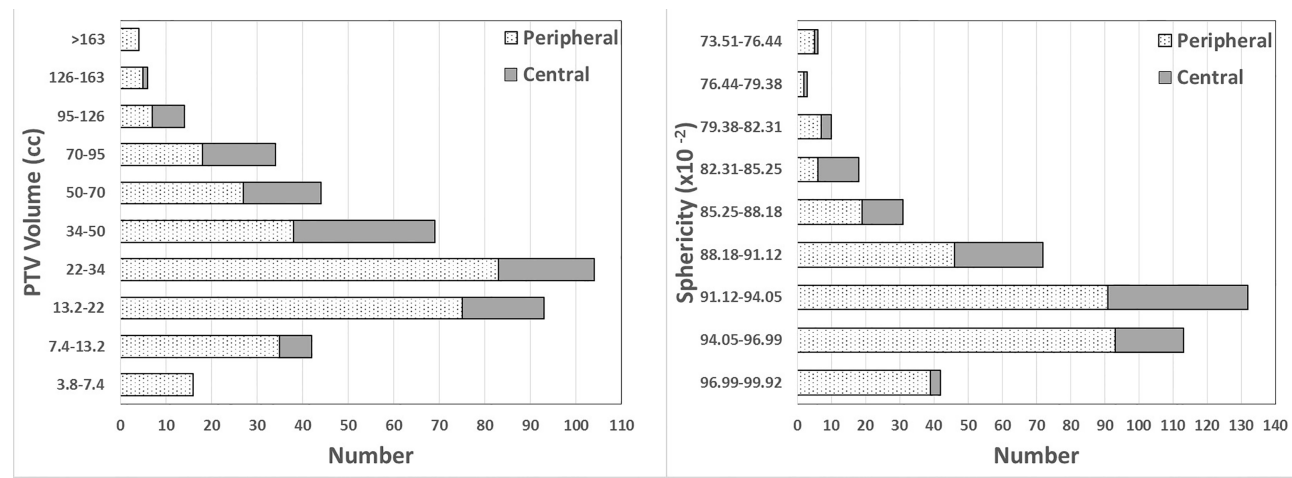

FIGURE 1 | Number distribution of PTV volumes and sphericity in this study. PTV volume is presented using the RTOG 0813 and 0915 volume bins. Sphericity is shown using bins equally spaced according to the sphericity range in this study.

\section{Results of Bivariate Analysis}

Table 3 presents the $\mathrm{R}^{2}$ of different fitting equations and the $\mathrm{p}$ value of two independent variables in the dependence analysis of GM on bivariaty $V_{P}$ and $S p$. The power equation obtained the highest $\mathrm{R}^{2}$, and the expressions of peripheral and central lesions are Equations 9 and 10, respectively.

$$
G M=0.676 V_{P}^{0.175} S p^{-0.069}
$$

with standard errors of $0.011,0.009$, and 0.128 for the three parameters.

$$
G M=0.662 V_{P}^{0.181} S p^{-0.100}
$$

with standard errors of $0.027,0.019$, and 0.259 for the three parameters.
The $\mathrm{R}^{2}$ of bivariate power equations for peripheral and central lesions was 0.675 and 0.527 , respectively, almost equal to fitting results with $\mathrm{V}_{\mathrm{P}}$ as the independent variable (0.675 and 0.526$)$. Compared with the equation of $G M$ versus $V_{P}$, the bivariate equation does not improve the fitting goodness. In addition, the $\mathrm{p}$-value of the power equations for peripheral and central lesions were less than 0.001 , while the $\mathrm{p}$-values of the variable $\mathrm{Sp}$ were greater than 0.05 ( 0.590 for peripheral and 0.494 for central), indicating that the original hypothesis that the variable $S p$ was zero could not be rejected. In the bivariate equation, Sp could hardly explain the GM, and these equations were unreliable.

\section{Results of the Equation Verification}

Using external independent validation sets, Table 4 lists the maximum, minimum, mean error, and standard deviation

\begin{tabular}{|c|c|c|c|c|c|c|c|c|c|c|c|}
\hline Volume bin (cc) & Tumor type & $\mathbf{N}$ & $V_{P}(c c)$ & $\operatorname{Sp}\left(\times 10^{-2}\right)$ & Actual GM (cm) & Analytic GM (cm) & $\mathbf{R} 50 \%$ & Cl & Rx dose (Gy) & Fractions & IMRT Factor \\
\hline \multirow[t]{2}{*}{$3.8-7.4$} & Peripheral & 15 & 6.25 & 98.20 & 0.92 & 0.93 & 6.03 & 0.81 & 48.67 & 4.13 & 2.24 \\
\hline & Central & 0 & - & - & - & & & - & - & - & - \\
\hline \multirow[t]{2}{*}{$7.4-13.2$} & Peripheral & 35 & 10.32 & 95.81 & 1.04 & 1.02 & 5.62 & 0.84 & 48.69 & 4.31 & 2.12 \\
\hline & Central & 7 & 9.67 & 94.77 & 1.02 & 1.00 & 5.48 & 0.85 & 55.71 & 7.29 & 1.99 \\
\hline \multirow[t]{2}{*}{$13.2-22$} & Peripheral & 75 & 18.37 & 93.91 & 1.13 & 1.13 & 4.80 & 0.86 & 47.52 & 4.59 & 2.08 \\
\hline & Central & 18 & 17.57 & 93.19 & 1.11 & 1.12 & 4.86 & 0.85 & 56.33 & 7.44 & 2.03 \\
\hline \multirow[t]{2}{*}{$22-34$} & Peripheral & 83 & 26.20 & 92.31 & 1.20 & 1.20 & 4.47 & 0.87 & 47.35 & 4.64 & 2.03 \\
\hline & Central & 21 & 28.68 & 92.14 & 1.20 & 1.23 & 4.34 & 0.87 & 54.00 & 7.05 & 1.94 \\
\hline \multirow[t]{2}{*}{$34-50$} & Peripheral & 38 & 41.51 & 90.83 & 1.31 & 1.31 & 4.21 & 0.86 & 44.42 & 4.63 & 2.02 \\
\hline & Central & 31 & 42.13 & 90.28 & 1.34 & 1.32 & 4.31 & 0.81 & 53.81 & 7.03 & 1.90 \\
\hline \multirow[t]{2}{*}{$50-70$} & Peripheral & 27 & 58.49 & 89.79 & 1.39 & 1.39 & 3.86 & 0.85 & 41.63 & 4.59 & 1.91 \\
\hline & Central & 17 & 59.94 & 89.21 & 1.44 & 1.41 & 4.37 & 0.86 & 52.24 & 6.82 & 1.93 \\
\hline \multirow[t]{2}{*}{$70-95$} & Peripheral & 18 & 79.01 & 89.22 & 1.55 & 1.47 & 4.06 & 0.88 & 36.78 & 4.33 & 1.87 \\
\hline & Central & 16 & 81.75 & 87.75 & 1.55 & 1.49 & 3.94 & 0.87 & 54.00 & 7.06 & 2.02 \\
\hline \multirow[t]{2}{*}{$95-126$} & Peripheral & 7 & 108.61 & 90.51 & 1.61 & 1.55 & 3.65 & 0.88 & 37.71 & 4.43 & 1.87 \\
\hline & Central & 7 & 105.03 & 87.58 & 1.47 & 1.56 & 3.89 & 0.87 & 50.57 & 6.57 & 1.89 \\
\hline \multirow[t]{2}{*}{$126-163$} & Peripheral & 5 & 146.95 & 86.07 & 1.58 & 1.64 & 3.49 & 0.87 & 38.00 & 4.60 & 1.71 \\
\hline & Central & 2 & 188.56 & 87.02 & 1.63 & 1.73 & 3.14 & 0.90 & 42.00 & 5.5 & 1.73 \\
\hline \multirow[t]{2}{*}{$>163$} & Peripheral & 4 & 212.09 & 83.94 & 1.58 & 1.74 & 2.92 & 0.88 & 27.00 & 3.50 & 2.14 \\
\hline & Central & 0 & - & - & - & & & - & - & - & - \\
\hline
\end{tabular}

TABLE 1 | Averages of the data for all lung SBRT treatment plans.

IMRT factor is the quotient of fractional monitor units and fractional dose in cGy.

$N$, the number of cases; $V_{P}$, PTV volume; Sp, sphericity; GM, gradient measure; Cl, conformity index (the quotient of the PTV volume receiving the prescription dose and the PTV volume); $R x$ dose, prescription dose. 
TABLE 2 | Fitting results of GM versus a univariaty $\left(V_{P}, S p\right)$ using different functional forms.

\begin{tabular}{|c|c|c|c|c|c|c|c|c|}
\hline \multirow[t]{3}{*}{ Equation } & \multicolumn{4}{|c|}{ Peripheral } & \multicolumn{4}{|c|}{ Central } \\
\hline & \multicolumn{2}{|c|}{$V p$} & \multicolumn{2}{|c|}{ Sp } & \multicolumn{2}{|c|}{$V p$} & \multicolumn{2}{|c|}{$\mathrm{Sp}$} \\
\hline & $\mathrm{R}^{2}$ & $p-v_{p}$ & $\mathrm{R}^{2}$ & $\mathrm{p}-\mathrm{Sp}$ & $\mathrm{R}^{2}$ & $\mathrm{p}-\mathrm{v}_{\mathrm{p}}$ & $\mathbf{R}^{2}$ & $p-S p$ \\
\hline Linear & 0.500 & $p<0.001$ & 0.229 & $p<0.001$ & 0.388 & $p<0.001$ & 0.150 & $p<0.001$ \\
\hline Logarithmic & 0.667 & $p<0.001$ & 0.222 & $p<0.001$ & 0.521 & $p<0.001$ & 0.143 & $p<0.001$ \\
\hline Exponential & 0.467 & $p<0.001$ & 0.239 & $p<0.001$ & 0.378 & $p<0.001$ & 0.152 & $p<0.001$ \\
\hline
\end{tabular}

$V_{P}$, PTV volume; Sp, sphericity; GM, gradient measure.

between analytical GM and actual GM for peripheral and central lung cancer. The mean absolute errors were 0.017 and $0.023 \mathrm{~cm}$ for peripheral and central lung cancer, respectively. We can see that both mean absolute errors are less than $0.03 \mathrm{~cm}$, and the standard deviation was slight (about $0.01 \mathrm{~cm}$ ).

\section{DISCUSSION}

This study analyzed the dependence of the GM on the PTV volume and shape (sphericity) for peripheral and central lung SBRT plans using the univariate and bivariate least-squares regressions. This study has demonstrated a predictable power equation between the GM of our center's clinically acceptable lung SBRT plans and the PTV volume. The equational correlation coefficient of the peripheral lesions is higher than that of central lesions. Unexpectedly, the correlation between the GM and the PTV shape was very low for two types of lung SBRT plans. Overall, to the best of our knowledge, few studies were available on the shape dependence of dose gradient for lung SBRT plans. Moreover, few scholars classified and compared peripheral and central lesions in other similar research. This work defined the dependence of the gradient measure of lung SBRT plan on the PTV volume and shape. The results can predict the GM before planning and then set up the shell (pseudo structure) used for optimization individually to reduce possible GM increase and the number of trials and errors. In addition, the fitting equation obtained in this study can be used as a primary tool to evaluate the dose gradient after the planning process to assist the dosimetric quality control.

In a study by Hoffman et al. (7) on peripheral lung SBRT plans, although the factors of target shape and tumor type
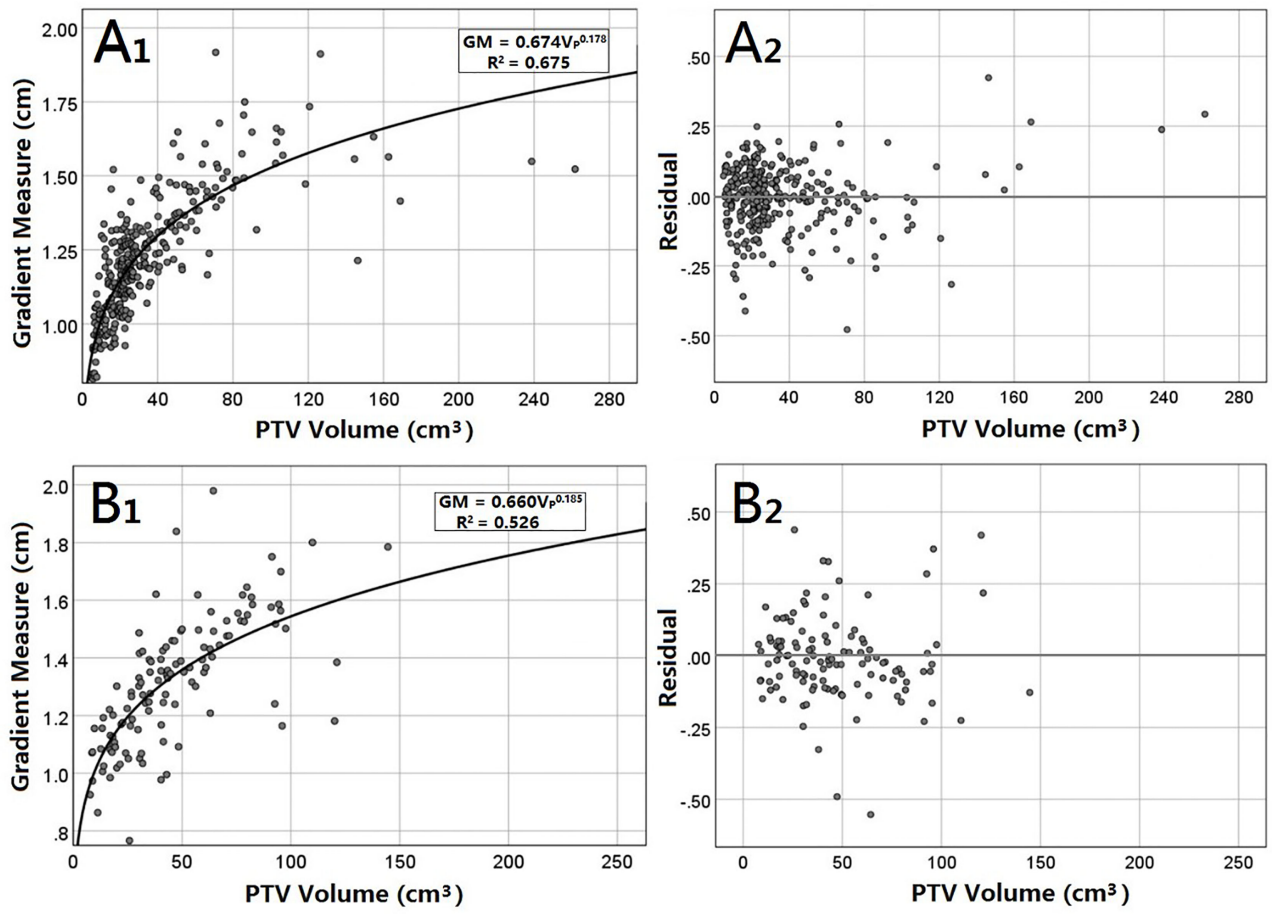

FIGURE 2 | GM versus the $V_{P}$ for peripheral $(\mathbf{A})$ and central (B) lung SBRT plans. The least-squares fit of the power equations $\left(\mathbf{A}_{\mathbf{1}}\right.$, $\left.\mathbf{B}_{\mathbf{1}}\right)$ is presented along with $\mathrm{R}^{2}$. In addition, residuals of the analytic GM minus the clinical GM are also presented $\left(\mathbf{B}_{\mathbf{1}}, \mathbf{B}_{\mathbf{2}}\right)$. 


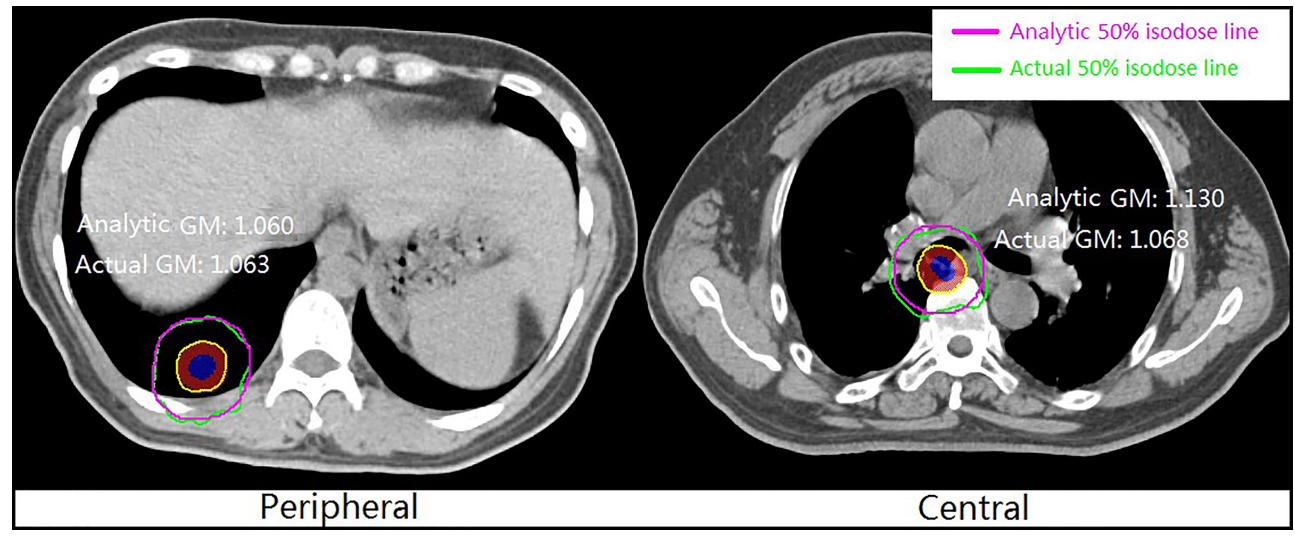

FIGURE 3 | Example of analytic and clinical 50\% isodose lines for peripheral and central lung SBRT plans.

(peripheral or central) were not considered, their conclusions were similar to part results of this work. They also found that the dependence of the GM on the PTV volume presented a power relationship, and the functional form of that relationship was $\mathrm{GM}=0.564 \mathrm{~V}_{P}^{0.215}$ with a standard error of 0.017 and 0.006 for the two parameters and an $\mathrm{R}^{2}$ value of 0.850 . The difference in $\mathrm{R}^{2}$ may be caused by data differences from two centers, such as the volume and range of the PTV, the treatment machine, the planning techniques, TPS, optimization methods, algorithms, etc. However, both we and Hoffman et al. found that the power function is the best to explain the relationship between the PTV volume and dose gradient. They got a slightly smaller GM (steeper dose fall-off) than this study (Eq. 8), which may be due to the data from different centers. We recalculated the GM of our enrolled cases using the results of Hoffman et al.'s study (Eq. 11), finding that the maximum and average analytic GM differences between the two studies were 0.10 and $0.063 \mathrm{~cm}$, respectively. This shows that our results are almost consistent with those of Hoffman et al. Some other studies have also concluded a positive correlation between dose gradient and target volume $(7,13,14)$, which agrees with our results.

The dependence of the GM on $V_{P}$ for peripheral lesions showed a higher $\mathrm{R}^{2}$ than that for central lesions ( 0.675 vs. 0.527$)$, indicating that compared with central lesions, the power function can better explain peripheral lesions. In other words, the GM of peripheral lesions has higher dependence on $V_{P}$ than that of central lesions. This is probably because the positional relationship between the target and OARs is more complex for central lesions. In order to meet the dosimetric constraints, the

TABLE 3 | Fitting results of GM on bivariaty $\left(V_{P}\right.$ and $\left.S p\right)$ for different equations.

\begin{tabular}{|c|c|c|c|c|c|c|}
\hline \multirow[t]{2}{*}{ Equation } & \multicolumn{3}{|c|}{ Peripheral } & \multicolumn{3}{|c|}{ Central } \\
\hline & $\mathbf{R}^{2}$ & $p-V_{p}$ & p-Sp & $\mathbf{R}^{2}$ & $p-V_{P}$ & $p-S p$ \\
\hline Linear & 0.523 & $p<0.001$ & $p<0.001$ & 0.400 & $p<0.001$ & $p<0.001$ \\
\hline Nonlinear sum & 0.668 & $p<0.001$ & 0.717 & 0.393 & $p<0.001$ & 0.324 \\
\hline Logarithmic & 0.667 & $p<0.001$ & 0.759 & 0.521 & $p<0.001$ & 0.630 \\
\hline Exponential & 0.498 & $p<0.001$ & $p<0.001$ & 0.521 & $p<0.001$ & $p<0.001$ \\
\hline Power & 0.675 & $p<0.001$ & 0.590 & 0.527 & $p<0.001$ & 0.494 \\
\hline
\end{tabular}

planning parameters (such as the beam settings) of the central lung cancer are more diversified, which reduced the regularity of the dose gradient, leading to low dependence of the GM on the PTV volume. In addition, 7 of the 307 peripheral lung SBRT plans had a PTV volume greater than $125 \mathrm{~cm}^{3}$. The residual (Figure 2 $\mathbf{A}_{\mathbf{2}}$ ) shows that when the PTV volume is greater than $125 \mathrm{~cm}^{3}$, Equation 8 for peripheral lung cancer will predict a higher GM, which is a limitation of the fitting results.

This study also investigated the dependence of the GM on the target shape (Table 1). However, for peripheral and central lung cancer, there were low correlation coefficients of the GM on shape variable $\mathrm{Sp}$ for all functional forms $\left(\mathrm{R}^{2}<0.25\right)$, and the corresponding equations were not acceptable. Similar to the univariate results, the power-function form of the GM on bivariaty also got the highest $\mathrm{R}^{2}$ (Table 2). However, compared with the results from the univariate analysis $V_{P}$, the $R^{2}$ of bivariate results was not improved for two types of lung cancer. It demonstrates that the bivariaty $\left(\mathrm{V}_{\mathrm{P}}\right.$ and $\left.\mathrm{Sp}\right)$ equations have the same explanatory power to the response variable $\mathrm{GM}$ as that of univariaty $\left(\mathrm{V}_{\mathrm{P}}\right)$. Moreover, the $\mathrm{p}$ values of $\mathrm{Sp}$ in the bivariate power functions were greater than 0.05 , which indicates that the contribution of $\mathrm{Sp}$ to the equation is unreliable. Therefore, the bivariate equations serve no practical purpose. All those results proved that the GM has no dependence on the PTV shape.

There are few studies on the dependence of dose gradient on the target shape for lung SBRT plans, and the relationship between them has not been determined. This study provides definitive evidence proving that the dose gradient has no dependence on the target shape, which may be explained as the target volume suitable for SBRT is usually small, resulting in small shape ranges. Moreover, with radiotherapy technology advances, targets with different shapes can easily achieve high conformity in SBRT plans. These factors make the shape have little effect on the dose gradient.

Overall, only the power equation of GM versus $V_{P}$ is reliable and acceptable for two types of lung cancer. The validation results using independent external data show that the mean absolute error of the GM for peripheral and central lung SBRT 
TABLE 4 | Absolute error of analytical and actual GM.

\begin{tabular}{lcc}
\hline GM (cm) & Peripheral & Central \\
\hline Maximum & 0.040 & 0.047 \\
Minimum & $<0.001$ & $<0.001$ \\
Mean & 0.017 & 0.023 \\
Std & 0.012 & 0.013 \\
\hline
\end{tabular}

was less than 0.2 and $0.3 \mathrm{~mm}$, respectively, which indicates that the GM's final fitting formula is relatively reliable.

The results of this work can be applied to create a shell (pseudo-structure) to minimize the GM and hence achieve a sharper dose gradient during the clinical planning process. Since the $\mathrm{CI}$ is near unity for most SBRT treatment plans at our center, the average distance from the $50 \%$ isodose line to the edge of the PTV is approximately the GM. The results of this study can be used to develop the following possible workflow: 1) The GM is calculated using Equation 8 or 9 individually for patients using a prewritten script; 2) The planner creates a shell (15) (see Figure 4 for details) with a distance of GM cm from the edge of PTV; 3) Before the optimization process, the maximum dose of the shell is set to lower than $50 \%$ prescription dose, such as $40 \%$, to reduce the GM as much as possible; 4) After the preliminary plan, the planner benchmarks the plan against the GM. As part of plan quality control (QC), the shell from Equation 8 or 9 may be used to determine how the plan performed relative to the plans in the dataset. If the $50 \%$ isodose radius exceeds the shell, the plan may need to be adjusted by changing the constraint of the shell to achieve a possible lower GM. Naturally, the plan should finally meet the RTOG 0813 and RTOG 0915 dosimetric constraints.

It should be noted that, for safety reasons, the treatment of patients whose OARs in the SBRT plan do not meet the RTOG 0813 and 0915 guidelines will not be allowed in our center, but the alternatives will be considered. In other institutions, there may be other solutions to this situation. The guidelines of RTOG 0813 (16) and RTOG 0915 (17) recommend that the maximum dose at $2 \mathrm{~cm}$ from PTV in any direction should range from $50 \%$ to $77 \%$ of the prescription dose according to different PTV volumes. In this study, the analytic distances between $50 \%$ isodose lines and the PTV of the total 426 enrolled cases obtained by Equations 8 and 9 were less than $2 \mathrm{~cm}$, which indicates that the analytic dose gradient is a more stringent constrain than $\mathrm{D}_{2 \mathrm{~cm}}(\mathrm{~Gy})$, which is suggested in the RTOG proposal and clinically achievable. In clinical practice, when the plan cannot meet the analytic results of Equations 8 and 9, doctors and planners should comprehensively evaluate the plan according to the guidelines such as RTOG and decide whether the plan can be applied to clinical treatment.

Here are some limitations and prospects of this study. This study did not include the impact of techniques (3D-CRT, IMRT, Arc), the number of beams, prescription dose, etc., on dose gradients. The impact of these factors on the results of this study requires additional data to explore $(14,20)$. In addition, the delivery machine used for this study was the Edge accelerator. The conclusion may be different if other equipment is used. The GM and target geometry relationship on other machines must be reexamined using methods similar to this study or other measures based on specific system characteristics. Finally, the obtained Equation 9 will overestimate the GM of the peripheral lung SBRT plan with PTV greater than $125 \mathrm{~cm}^{3}$. A separate equation could fit the large PTV data, but this requires more treatment plans in this volume range.

\section{CONCLUSION}

The gradient measure of the lung SBRT plan is shapeindependent but volume-dependent. The dependence of the GM on the PTV volume for peripheral and central lung cancer can be described by two different power equations, and the correlation is higher for peripheral lesions. The results of this

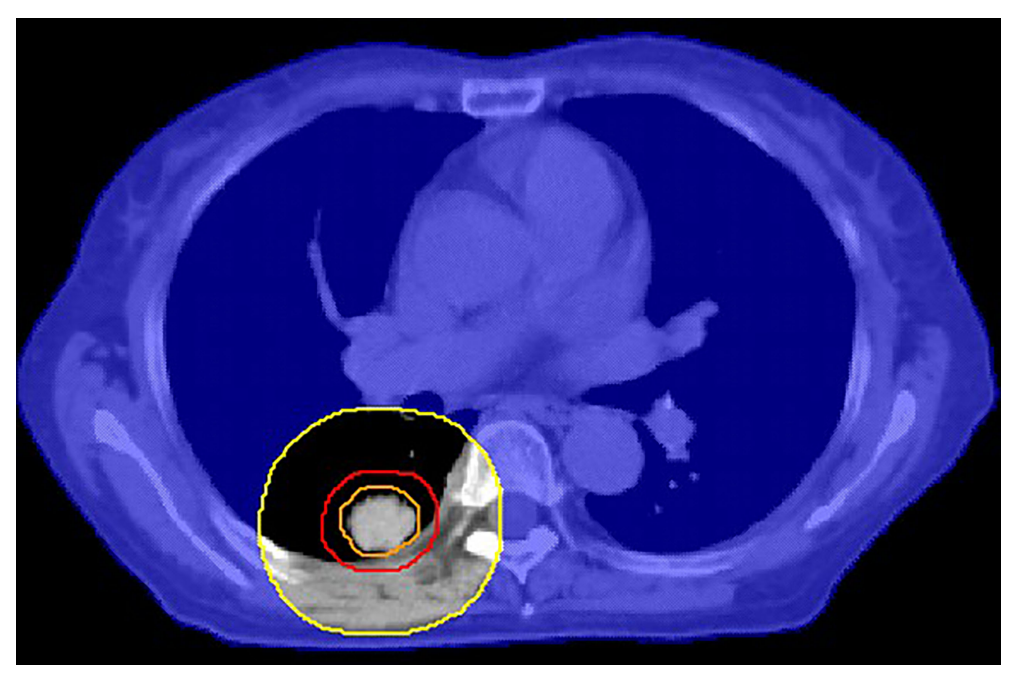

FIGURE 4 | An example of a cross-sectional view of the shell (orange: ITV, red: PTV, yellow: auxiliary structure after GM cm expansion of PTV, blue shade: GM cm shell obtained by body minus auxiliary structure). This shell is used to control the $50 \%$ isodose line. 
study can be used for preplan prediction and postplan review of gradient measure and serve as a potential tool to assist dosimetric quality control during the radiosurgery process.

\section{DATA AVAILABILITY STATEMENT}

The raw data supporting the conclusions of this article will be made available by the authors, without undue reservation.

\section{ETHICS STATEMENT}

The approved lung SBRT treatment plans in Shanghai Chest Hospital were retrospectively selected. When the study began, all selected patients signed informed consents and completed radiotherapy. Ethical standards and patients' confidentiality were ensured and in line with regulations of the local institutional review board and data safety laws. This study was approved by the Ethics Committee of Shanghai Chest Hospital (the committee's reference Number: KS1863).

\section{REFERENCES}

1. Chang JY, Senan S, Paul MA, Mehran RJ, Louie AV, Balter P, et al. Stereotactic Ablative Radiotherapy Versus Lobectomy for Operable Stage I non-Small-Cell Lung Cancer: A Pooled Analysis of Two Randomised Trials. Lancet Oncol (2015) 16(6):630-7. doi: 10.1016/S1470-2045(15)70168-3

2. Onishi H, Shirato H, Nagata Y, Hiraoka M, Fujino M, Gomi K, et al. Stereotactic Body Radiotherapy (SBRT) for Operable Stage I non-SmallCell Lung Cancer: Can SBRT be Comparable to Surgery ? Int J Radiat Oncol Biol Phys (2011) 81(5):1352-8. doi: 10.1016/j.ijrobp.2009.07.1751

3. Murray L, Ramasamy S, Lilley J, Snee M, Clarke K, Musunuru HB, et al. Stereotactic Ablative Radiotherapy (SABR) in Patients With Medically Inoperable Peripheral Early Stage Lung Cancer: Outcomes for the First UK SABR Cohort. Clin Oncol (2016) 28(1):4-12. doi: 10.1016/j.clon.2015.09.007

4. Takeda A, Kunieda E, Ohashi T, Aoki Y, Koike N, Takeda T. Stereotactic Body Radiotherapy (SBRT) for Oligometastatic Lung Tumors From Colorectal Cancer and Other Primary Cancers in Comparison With Primary Lung Cancer. Radiotherapy Oncol (2011) 101(2):255-9. doi: 10.1016/ j.radonc.2011.05.033

5. Chen G, Dong B, Shan G, Zhang X, Tang H, Li Y, et al. Choice of Immobilization of Stereotactic Body Radiotherapy in Lung Tumor Patient by BMI. BMC Cancer (2019) 19(1):583. doi: 10.1186/s12885-019-5767-1

6. Uematsu M, Shioda A, Suda A, Fukui T, Ozeki Y, Hama Y, et al. Computed Tomography-Guided Frameless Stereotactic Radiotherapy for Stage I nonSmall Cell Lung Cancer: A 5-Year Experience. Int J Radiat Oncol Biol Phys (2001) 51(3):666-70. doi: 10.1016/s0360-3016(01)01703-5.

7. Hoffman D, Dragojević I, Hoisak J, Hoopes D, Manger R. Lung Stereotactic Body Radiation Therapy (SBRT) Dose Gradient and PTV Volume: A Retrospective Multi-Center Analysis. Radiat Oncol (2019) 14(1):1-7. doi: 10.1186/s13014-019-1334-9

8. Videtic GM, Hu C, Singh AK, Chang JY, Parker W, Olivier KR, et al. A Randomized Phase II Study Comparing 2 Stereotactic Body Radiation Therapy (SBRT) Schedules for Medically Inoperable Patients With Stage I Peripheral non-Small Cell Lung Cancer: NRG Oncology RTOG 0915 (NCCTG N0927). Int J Radiat Oncol Biol Phys (2012) 93(4):757-64. doi: 10.1016/j.ijrobp.2015.07.2260

9. Desai DD, Johnson EL, Cordrey IL. An Analytical Expression for R50\% Dependent on PTV Surface Area and Volume: A Lung SBRT Comparison. J Appl Clin Med Phys (2020) 21(11):278-82. doi: 10.1002/acm2.13026

\section{AUTHOR CONTRIBUTIONS}

YD: Study concepts, study design, data acquisition, statistical analysis, manuscript preparation, and manuscript editing. YL: Data acquisition, quality control of data and algorithms, and data analysis and interpretation. HW: Study design, statistical analysis, and manuscript review. BK: Quality control of data and algorithms and data analysis and interpretation. AF: Study concepts and manuscript review. KM: Quality control of data and algorithms. HC, YH, HG, and YS: Manuscript review. TZ: Study concepts and manuscript review. QK: Study concepts, study design, manuscript review, and wrote the paper. ZX: Study concepts, study design, and manuscript review. All authors contributed to the article and approved the submitted version.

\section{FUNDING}

The authors thank the Nurture projects for basic research of Shanghai Chest Hospital (No. 2019YNJCM05) for its financial support.

10. Eclipse Photon and Electron 15.5 Reference Guide. Varian Med Syst (2017), $1-575$.

11. Desai DD, Cordrey IL, Johnson EL. A Physically Meaningful Relationship Between R50\% and PTV Surface Area in Lung SBRT. J Appl Clin Med Phys (2020) 21(9):47-56. doi: 10.1002/acm2.12964

12. Desai D, Srinivasan S, Elasmar H, Johnson E. SU-E-T-78: A Study of Dose Falloff Gradient in RapidArc Planning of Lung SBRT. Med Phys (2015) 42 (6Part13):3349-9. doi: 110.1118/1.4924439

13. Delgado AB, Cohen D, Eng TY, Stanley DN, Shi Z, Charlton M, et al. Modeling the Target Dose Fall-Off in IMRT and VMAT Planning Techniques for Cervical SBRT. Med Dosimetry (2018) 43(1):1-10. doi: 110.1016/j.meddos

14. Narayanasamy G, Desai D, Morrill S, Zhang X, Galhardo E, Maraboyinay S, et al. Technical Note: A Planning Technique to Lower Normal Tissue Toxicity in Lung SBRT Plans Based on Two Likely Dependent RTOG Metrics. Med Phys (2018) 45(5):2325-8. doi: 10.1002/mp.12833

15. Duan Y, Gan W, Wang H, Chen H, Gu H, Shao Y, et al. On the Optimal Number of Dose-Limiting Shells in the SBRT Auto-Planning Design for Peripheral Lung Cancer. J Appl Clin Med Phys (2020) 21(9):134-42. doi: $10.1002 / \mathrm{acm} 2.12983$

16. Bezjak A, Paulus R, Gaspar JE, Timmerman RD, Straube WL, Ryan WF, et al. Radiation Therapy Oncology Group RTOG 0813 Seamless Phase I/II Study of Stereotactic Lung Radiotherapy (SBRT) For Early Stage, Centrally Located, non-Small Cell Lung Cancer (NSCLC) in Medically Inoperable Patients. (2019). 37(15):1316-1325. doi: 10.1200/JCO.18.00622

17. Videtic GM, Paulus R, Singh AK, Chang JY, Parker W, Olivier KR, et al. Radiation Therapy Oncology Group (RTOG) Protocol 0915: A Randomized Phase 2 Study Comparing 2 Stereotactic Body Radiation Therapy (SBRT) Schedules for Medically Inoperable Patients With Stage I Peripheral nonSmall Cell Lung Cancer. Int J Radiat Oncol Biol Phys (2013) 87:S3. doi: 10.1016/j.ijrobp.2013.06.016

18. Treuer H, Hoevels M, Luyken K, Visser-Vandewalle V, Wirths J, Kocher $M$, et al. Intracranial Stereotactic Radiosurgery With an Adapted Linear Accelerator vs. Robotic Radiosurgery: Comparison of Dosimetric Treatment Plan Quality. Strahlenther Onkol (2015) 191(6):470-6. doi: 10.1007/s00066-014-0786-y

19. Suddick DE. Chance R2 Estimate of Overfit. J Exp Educ (1974) 42(3):93-6. doi: 10.1080/00220973.1974.11011484

20. Narayanasamy G, Desai D, Maraboyina S, Penagaricano J, Zwicker R, Johnson EL. A Dose Fall-Off Gradient Study in RapidArc Planning of Lung Stereotactic 
Body Radiation Therapy. J Med Phys (2018) 43(3):147-157. doi: 10.4103/ jmp.JMP_38_18

Conflict of Interest: BK was employed by MIM Software Inc., and KM was employed by Varian Medical Systems.

The remaining authors declare that the research was conducted in the absence of any commercial or financial relationships that could be construed as a potential confict of interest.

The authors declare that this study received funding from Nurture projects for basic research of Shanghai Chest Hospital (No. 2019YNJCM05). The funder was not involved in the study design, collection, analysis, and interpretation of data, the writing of this article or the decision to submit it for publication.
Publisher's Note: All claims expressed in this article are solely those of the authors and do not necessarily represent those of their affiliated organizations, or those of the publisher, the editors and the reviewers. Any product that may be evaluated in this article, or claim that may be made by its manufacturer, is not guaranteed or endorsed by the publisher.

Copyright $\odot 2021$ Duan, Lin, Wang, Kang, Feng, Ma, Chen, Huang, Gu, Shao, Zhou, Kong and $X u$. This is an open-access article distributed under the terms of the Creative Commons Attribution License (CC BY). The use, distribution or reproduction in other forums is permitted, provided the original author(s) and the copyright owner(s) are credited and that the original publication in this journal is cited, in accordance with accepted academic practice. No use, distribution or reproduction is permitted which does not comply with these terms. 